\title{
A Snapshot of Compliance with the Sepsis Six Care Bundle in Two Acute Hospitals in the West Midlands, UK
}

\author{
Catriona Frankling ${ }^{1}$, Jaimin Patel $^{2}$, Ben Sharif ${ }^{3}$, Teresa Melody ${ }^{4}$, Joyce Yeung ${ }^{5}$, Fang Gao ${ }^{6}$, Tamas Szakmany ${ }^{7}$
}

\begin{abstract}
Background:The sepsis six care bundle has been adopted by hospitals in England and Wales for the management of patients with sepsis, with the aim of increasing survival when all elements of the bundle are achieved.

Aim: To assess compliance with the Sepsis Six Care Bundle in two acute NHS hospitals in the West Midlands.

Materials and Methods: Adults admitted to hospital over a 24-hour period were screened for sepsis. Sepsis was identified using the Systemic Inflammatory Response (SIRS) criteria and the quick sequential organ failure assessment (qSOFA) score. Adherence to the Sepsis Six Care Bundle was assessed.

Results: 249 patients were screened and 24 patients were identified as having sepsis (9.6\%). One patient received all six elements of the bundle. Compliance was highest for giving intravenous fluids (58.3\%) and antibiotics (58.3\%), and lowest for measuring urine output (16.7\%).

Conclusions: Further research is needed to establish the reasons for low compliance.

Indian Journal of Critical Care Medicine (2019): 10.5005/jp-journals-10071-23204
\end{abstract}

\section{INTRODUCTION}

Sepsis is a life-threatening organ dysfunction caused by a dysregulated host response to infection. ' It is estimated that 44,100 deaths per year are attributable to sepsis in the UK, costing the NHS a projected $£ 7.76$ billion. ${ }^{2}$ The incidence of sepsis continues to rise and has been ascribed to an ageing population with multiple co-morbidities, as well as an increase in the recognition of sepsis. ${ }^{3,4}$ It is estimated that sepsis is now a leading cause of mortality and critical illness across the world. ${ }^{3,4}$

Survival from sepsis may be improved when it is recognised and treated promptly. ${ }^{5}$ In an attempt to improve outcomes from sepsis hospitals in England and Wales have adopted the use of the Sepsis Six Care Bundle (Table 1). ${ }^{5}$

Care bundles were developed by the Institute for Health Care Improvement (IHI) and are small collections of evidencebased tasks, that when implemented together should achieve better outcomes than when instigated individually. ${ }^{6}$ Since the establishment of the sepsis six care bundle in 2007, there has been limited data on compliance rates with the bundle, and studies related to the impact of the care bundle on mortality rates show conflicting results. An observational study conducted in 2007-2008 when the bundle was initially established showed that $36.6 \%$ of patients with severe sepsis received the bundle, with a mortality rate of $20 \%$, compared to $44.1 \%$ for patients who did not receive the care bundle. ${ }^{5}$ However, a recent study in Wales found that only $12 \%$ of 290 patients with sepsis received the full sepsis six care bundle, yet there was no significant difference in mortality related to delivery of the Bundle.

The Parliamentary and Health Service Ombudsman and National Confidential Enquiry into Patient Outcome and Death (NCEPOD) reports both recommend audit of compliance with sepsis care bundles. ${ }^{8,9}$ It is important to assess compliance with the Sepsis Six Care Bundle to highlight any barriers to good practice, and assess the impact this has on patient outcomes.

The aim of this study was to provide a snapshot of compliance to the sepsis six care bundle in two acute hospitals in the West Midlands.

\begin{abstract}
1,2,6 Institute of Inflammation and Ageing, College of Medical and Dental Sciences, University of Birmingham, Birmingham, UK

3,7Department of Anesthesia, Intensive Care and Pain Medicine, Division of Population Medicine, Cardiff University, Cardiff, UK

${ }^{4}$ Academic Department of Anesthesia, Critical Care, Resuscitation and Pain, Heart of England NHS Foundation Trust, Birmingham, UK

${ }^{5}$ Department of Warwick Clinical Trials Unit, Warwick Medical School, University of Warwick, Warwick, UK

Corresponding Author: Tamas Szakmany, Department of Anesthesia, Intensive Care and Pain Medicine, Division of Population Medicine, Cardiff University, Cardiff, UK, e-mail: szakmanyt1@cardiff.ac.uk

How to cite this article: Frankling C, Patel J, Sharif B, Melody T, Yeung J, Gao F, et al. A Snapshot of Compliance with the Sepsis Six Care Bundle in Two Acute Hospitals in the West Midlands, UK. Indian J Crit Care Med 2019;23(7):310-315.
\end{abstract}

Source of support: Nil

Conflict of interest: None

Table 1: The sepsis six care bundle ${ }^{5}$

Give high-flow oxygen via non- Take blood cultures and consider rebreathe bag source control

Give intravenous (IV) antibiotics Check lactate according to local protocol

Start IV fluid resuscitation e.g. Monitor hourly urine output and Hartmann's or equivalent consider catheterisation

\section{Methods}

The study was an assessment of compliance with a recognised standard of care and did not involve any study interventions or collection of patient identifiable data, therefore no ethical approval was required, as demonstrated by the Health Research Authority (HRA) decision tool. ${ }^{10}$ The study protocol was reviewed and approved by the local research and development departments of the participating hospital trusts. The protocol was discussed

(0) The Author(s). 2019 Open Access This article is distributed under the terms of the Creative Commons Attribution 4.0International License (https://creativecommons. org/licenses/by-nc/4.0/), which permits unrestricted use, distribution, and non-commercial reproduction in any medium, provided you give appropriate credit to the original author(s) and the source, provide a link to the Creative Commons license, and indicate if changes were made. The Creative Commons Public Domain Dedication waiver (http://creativecommons.org/publicdomain/zero/1.0/) applies to the data made available in this article, unless otherwise stated. 
Table 2: Modified early warning score (MEWS), courtesy of Birmingham Heartlands Hospital

\begin{tabular}{|c|c|c|c|c|c|c|c|}
\hline Score & 3 & 2 & 1 & 0 & 1 & 2 & 3 \\
\hline \multicolumn{8}{|l|}{ Categories } \\
\hline Respirations (breaths per minute) & & 8 or less & & $9-16$ & $17-20$ & $21-29$ & 30 or more \\
\hline Oxygen Saturations (\%) & & & & 94 or more & $90-93$ & $85-89$ & 84 or less \\
\hline Systolic Blood Pressure (mm Hg) & 70 or less & $71-80$ & $81-100$ & $101-199$ & & 200 or more & \\
\hline Pulse (beats per minute) & & & & $51-100$ & $101-110$ & $111-129$ & 130 or more \\
\hline Conscious Level & & & $\begin{array}{l}\text { New Confusion/ } \\
\text { Agitation }\end{array}$ & Alert & $\begin{array}{l}\text { Responds to } \\
\text { Voice }\end{array}$ & $\begin{array}{l}\text { Responds to } \\
\text { Pain }\end{array}$ & Unresponsive \\
\hline Temperature $\left({ }^{\circ} \mathrm{C}\right)$ & & 35 or less & $35.1-36$ & $36.1-37.5$ & $37.6-38.1$ & 38.2 or more & \\
\hline Urine (mL per hour) & & & & No concerns & $21-35$ & $1-20$ & Nil \\
\hline
\end{tabular}

Table 3: Standard early warning score (SEWS), courtesy of University Hospital Birmingham

\begin{tabular}{|c|c|c|c|c|c|c|c|}
\hline Score & 3 & 2 & 1 & 0 & 1 & 2 & 3 \\
\hline \multicolumn{8}{|l|}{ Category } \\
\hline Heart Rate (beats per minute) & $<30$ & $30-39$ & $40-49$ & $50-99$ & $100-109$ & $110-129$ & $\geq 130$ \\
\hline Systolic Blood Pressure $(\mathrm{mm} \mathrm{Hg})$ & & $70-79$ & $80-99$ & 100-199 & & $\geq 200$ & \\
\hline Oxygen Saturations (\%) & $<85$ & $85-89$ & $90-92$ & $\geq 93$ & & & \\
\hline $\begin{array}{l}\text { Respiratory Rate (breaths per } \\
\text { minute) }\end{array}$ & $<9$ & & & $9-20$ & $20-30$ & $31-35$ & $\geq 36$ \\
\hline Temperature $\left({ }^{\circ} \mathrm{C}\right)$ & $<34$ & 34 & 35 & $36-37$ & $>38$ & $\geq 39$ & \\
\hline Conscious level & & & & Alert & $\begin{array}{l}\text { Responds to } \\
\text { Voice }\end{array}$ & $\begin{array}{l}\text { Responds to } \\
\text { Pain }\end{array}$ & Unresponsive \\
\hline
\end{tabular}

Table 4: The systemic inflammatory response syndrome ${ }^{11}$

Two or more of:

- Temperature more than $38^{\circ} \mathrm{C}$ or less than $36^{\circ} \mathrm{C}$

- Heart rate more than 90 beats per minute

- Respiratory rate more than 20 breaths per minute or $\mathrm{PaCo}_{2}<32$ $\mathrm{mm} \mathrm{Hg}(4.3 \mathrm{kPa})$

- White blood cell count $>12000 / \mathrm{mm}^{3}$ or $<4000 / \mathrm{mm}^{3}$ or $>10 \%$ immature bands

- Altered mental state

- Hyperglycemia (plasma glucose $>7.7 \mathrm{mmol} / \mathrm{L}$ ) in the absence of diabetes

Table 5: The qSOFA score ${ }^{1}$

Two or more of:

- Respiratory rate of 22 breaths per minute or more

- Altered mentation

- Systolic blood pressure $\leq 100 \mathrm{~mm} \mathrm{Hg}$

with the local Patient and Public Involvement (PPI) group who scrutinised and approved the final study objective, design and outcome measures.

Data collection took place on 22nd June 2016 at two large University affiliated acute hospitals in the West Midlands (Birmingham Heartlands Hospital ( $\mathrm{BHH}, 692$ in-patient beds) and University Hospital Birmingham (UHB, 1215 in-patient beds). All acute admissions between 00:00 and 23:59 on 22nd June 2016 were eligible. Patients under the age of 18 were excluded. A team, independent of clinical delivery collected the data and all medical teams at each hospital site were informed of the study when data collection took place.

\section{Screening for Sepsis}

Patients were screened for a Modified or Standard Early Warning Score (MEWS or SEWS) of three or above using either electronic records or medical notes. MEWS is the scoring system used at $\mathrm{BHH}$ (Table 2), whilst SEWS is used at UHB (Table 3).

Patients with a MEWS or SEWS score of three or above were assessed for a high clinical suspicion of an infection by members of the study team (based upon clinical history, examination and investigations). The Systemic Inflammatory Response (SIRS) criteria for sepsis (Table 4) and the quick sequential organ failure assessment (qSOFA) score (Table 5) were used to screen for Sepsis.

Patients were deemed to have sepsis and were included for assessment of compliance with the Sepsis Six Care Bundle if they scored two or more on either the SIRS criteria, or the qSOFA score (Flowchart 1).

\section{Assessing Compliance}

For the purpose of the study, 'time zero' for implementing the bundle began when the MEWS or SEWS score was first recorded as three or more. Compliance was defined as implementation of all six steps of the Bundle within one hour from time zero. Compliance to individual elements was also documented at one hour and at any time point up until time of data collection.

\section{Data Collection}

Data was collected across the two hospitals via a secure opensource web-based toolkit on hand held electronic devices which were sourced from the Welsh Intensive Care Society. The toolkit was developed for use in a previous sepsis study which was conducted in Wales, ${ }^{12}$ and was adapted for our use. This study collected patient data from observational charts, medical notes and electronic records as appropriate. Data collected included basic patient characteristics, admission diagnoses, vital signs observations, MEWS or SEWS scores, laboratory values (including blood culture results), criteria used to confirm suspected sepsis and sepsis management. Length of stay and hospital mortality were also recorded. 


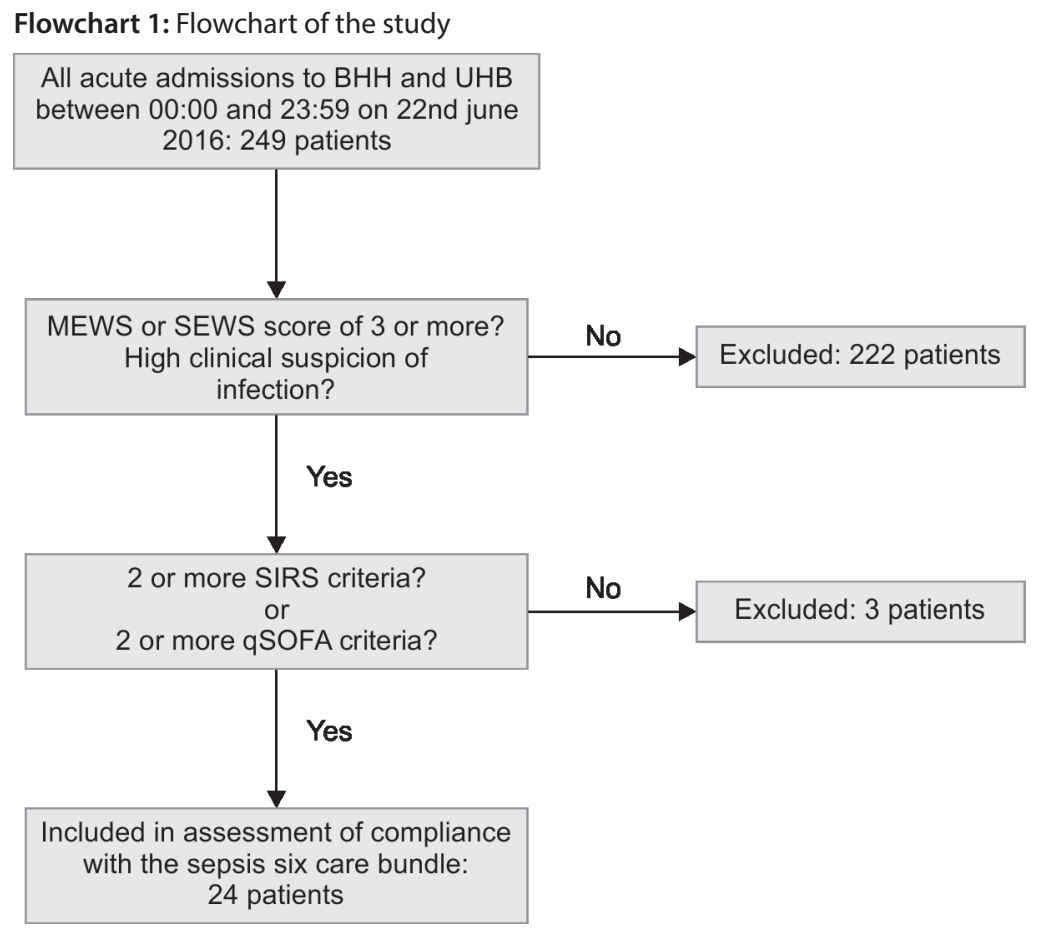

\section{Data Analysis}

Data was analysed using Excel version 14.0.6112.5000, Microsoft, USA and SPSS Statistics version 23, IBM. Descriptive statistics have been used. Data was tested for normality using a Shapiro-Wilk test. Categorical variables are described as proportions and mode. Measures for continuous variables are described using median and inter-quartile range (IQR).

\section{Results}

There were 249 acute adult admissions over the 24-hour study period and all of these patients were screened for suspected sepsis (Fig. 1). Ninety-eight patients were screened at UHB, with $10(10.2 \%)$ having a SEWS score of three or more. All 10 met the diagnostic criteria for sepsis. At $\mathrm{BHH}, 151$ patients were screened, with $17(11.2 \%)$ having a MEWS score of three or above. Of these, 14 (82.4\%) met the diagnostic criteria for sepsis as defined by our study (SIRS $\geq 2$ or qSOFA $\geq 2$ ). Overall, 24 patients (9.6\%) met the criteria for sepsis. All 24 patients met the SIRS diagnostic criteria for sepsis but only six (25\%) had a qSOFA score of two or above. There were no patients who met the qSOFA score alone without also meeting the SIRS criteria for sepsis.

Patient demographics can be viewed in Table 6 . The majority of patients were admitted from their homes (21 patients, 87.5\%) and were admitted under acute medicine (15 patients, $62.5 \%$ ) with care being delivered on Medical Assessment Units (16 patients, $66.7 \%$ ). Patients had a wide range of comorbidities, most commonly diabetes (six patients, 25.0\%), hypertension (seven patients, 29.2\%) and hypercholesterolaemia (seven patients, 29.2\%). Two patients (8.33\%) had a Do Not Attempt Resuscitation (DNAR) order and documented limitations on treatment.

The most common SIRS criteria that occurred in patients with suspected sepsis was a raised heart rate of more than 90 beats per minute (18 patients, $75 \%$ ) (Table 7 ). The most common qSOFA criteria that occurred was respiratory rate more than 22 breaths per minute (10 patients, $41.7 \%$ ).

The commonest suspected source of infection was pulmonary (10 patients, $41.7 \%$ ), followed by urinary tract (three patients, $12.5 \%$ ) and intra-abdominal (three patients, $12.5 \%$ ). Twenty patients (83.3\%) were not diagnosed with sepsis by the admitting team, including three patients (12.5\%) who were not identified by the admitting team with any form of infection.

The median MEWS or SEWS scores was four (IQR 3-5). Fifteen patients had a MEWS score of four or more, which mandates a review by critical care outreach as per hospital guidelines. However, only one of these 15 patients (6.67\%) was reviewed. This patient had a SEWS score of four. None of the patients were admitted to critical care or had any other critical care involvement.

Eight patients had blood cultures taken, and two were positive (Methicillin sensitive Staphylococcus aureus from one patient, Staphylococcus epidermidis and Actinomyces $\mathrm{sp}$ from another patient).

Only one patient had all aspects of the Sepsis Six Care Bundle completed (Table 8). For individual Bundle elements, compliance was highest for intravenous fluids (14 patients, 58.3\%) and intravenous antibiotics (14 patients, 58.3\%). Compliance was lowest for measuring urine output (four patients, 16.7\%). For the four patients with sepsis diagnosed by the team responsible for medical management, none received all elements of the Care Bundle, although all four patients received intravenous antibiotics. Three of the four patients diagnosed with sepsis by the admitting team were given intravenous fluids, two had blood cultures taken and one had a lactate measured. None were given oxygen and none had their urine output measured.

No patients died during their hospital admission. One (4.17\%) died within 30 days of admission and a further two patients (8.33\%) died within 60 days. All three were receiving palliative care for cancer. The median length of stay in hospital was 7.5 days (interquartile range 3-12 days). 
Table 6: Demographics of patients identified as having suspected sepsis

\begin{tabular}{|c|c|}
\hline Patient demographic & $\begin{array}{l}\text { Suspected sepsis patients } \\
(n=24)\end{array}$ \\
\hline Age (median [interquartile range]) & $62(47.8-77.5)$ \\
\hline Gender: male n (\%) & $14(58.3)$ \\
\hline \multicolumn{2}{|l|}{ Admission Source n (\%) } \\
\hline Home & $21(87.5)$ \\
\hline Other Hospital & $1(4.17)$ \\
\hline Nursing Home & $2(8.33)$ \\
\hline \multicolumn{2}{|l|}{ Specialty $n(\%)$} \\
\hline Acute Medicine & $15(62.5)$ \\
\hline General Surgery & $2(8.33)$ \\
\hline Respiratory & $2(8.33)$ \\
\hline Cardiothoracics & $2(8.33)$ \\
\hline Oncology & $1(4.17)$ \\
\hline Stroke & $1(4.17)$ \\
\hline Endocrine & $1(4.17)$ \\
\hline \multicolumn{2}{|l|}{ Ward n (\%) } \\
\hline Medical assessment unit & $16(66.7)$ \\
\hline Surgical assessment unit & $1(4.17)$ \\
\hline General medical & $4(16.7)$ \\
\hline General surgical & $2(8.33)$ \\
\hline \multicolumn{2}{|l|}{ Comorbidities n (\%) } \\
\hline Diabetes & $6(25.0)$ \\
\hline Heart Failure & $2(8.33)$ \\
\hline Hypertension & $7(29.2)$ \\
\hline Ischaemic heart disease & $4(16.7)$ \\
\hline Liver disease & $1(4.17)$ \\
\hline Recent chemotherapy & $2(8.33)$ \\
\hline Smoker & $4(16.7)$ \\
\hline Ex-smoker & $3(12.5)$ \\
\hline \multicolumn{2}{|l|}{ Drug History n (\%) } \\
\hline ACE-inhibitor & $3(12.5)$ \\
\hline Beta blocker & $2(8.33)$ \\
\hline Chronic antibiotics & $1(4.17)$ \\
\hline Diuretics & $6(25.0)$ \\
\hline Immunosuppressant & $2(8.33)$ \\
\hline Insulin & $4(16.7)$ \\
\hline HMG-CoA reductase inhibitors & $7(29.2)$ \\
\hline Steroids & $2(8.33)$ \\
\hline DNAR n (\%) & $2(8.70)$ \\
\hline Ceiling of treatment (ward) $n$ (\%) & $2(8.70)$ \\
\hline
\end{tabular}

\section{Discussion}

Our main finding was that despite the Sepsis Six Care Bundle being implemented for nearly a decade, compliance remains low. This was demonstrated in both hospitals, suggesting that the problem is unlikely to be due to local factors affecting just one individual hospital. As only four (16.7\%) patients were diagnosed with sepsis by the admitting team it is likely that lack of recognition is one reason for poor compliance. However, even the patients specifically labelled by the medical team as having sepsis were not managed as per the Sepsis Six Care Bundle. It is perhaps reassuring to see that compliance is highest for intravenous antibiotics and fluid administration, arguably the more important elements of the
Table 7: Infection characteristics of patients with suspected sepsis

\begin{tabular}{|c|c|}
\hline Infection Characteristics & $\begin{array}{l}\text { Suspected Sepsis } \\
\text { Patients }(n=24)\end{array}$ \\
\hline \multicolumn{2}{|l|}{ Source of Sepsis n (\%) } \\
\hline Pulmonary & $10(41.7)$ \\
\hline Urinary tract & $3(12.5)$ \\
\hline Intra-abdominal & $3(12.5)$ \\
\hline Indwelling vascular device & $2(8.33)$ \\
\hline Other & $2(8.33)$ \\
\hline Source unknown & $4(16.7)$ \\
\hline $\begin{array}{l}\text { MEWS/SEWS score median (interquartile } \\
\text { range) }\end{array}$ & $4(3-5)$ \\
\hline Two or more SIRS Criteria Present n (\%) & $24(100)$ \\
\hline \multicolumn{2}{|l|}{ Individual SIRS Criteria Present n (\%) } \\
\hline Temp $>38.3^{\circ} \mathrm{C}$ & $8(33.3)$ \\
\hline Temp $<36^{\circ} \mathrm{C}$ & $3(12.5)$ \\
\hline Altered mental state & $7(29.2)$ \\
\hline $\mathrm{HR}>90 /$ minute & $18(75.0)$ \\
\hline $\mathrm{RR}>20 /$ minute & $13(54.2)$ \\
\hline WCC $>12,000 / \mu \mathrm{L}$ & $15(62.5)$ \\
\hline $\mathrm{WCC}<4000 / \mu \mathrm{L}$ & $2(8.30)$ \\
\hline Glucose $>7.7 \mathrm{mmol} / \mathrm{L}$ & $7(29.2)$ \\
\hline Two or more qSOFA Criteria Present n (\%) & $6(25.0)$ \\
\hline \multicolumn{2}{|l|}{ Individual qSOFA Criteria Present n (\%) } \\
\hline $\mathrm{RR}>22 /$ minute & $10(41.7)$ \\
\hline Altered mentation & $7(29.2)$ \\
\hline Systolic BP $<100 \mathrm{~mm} \mathrm{Hg}$ & $7(29.2)$ \\
\hline Sepsis screening tool completed $\mathrm{n}(\%)$ & $2(8.33)$ \\
\hline Seen by Critical Care Outreach n (\%) & $1(4.17)$ \\
\hline In-hospital Mortality n (\%) & $0(0)$ \\
\hline 30-day Mortality n (\%) & $1(4.17)$ \\
\hline 60-day Mortality n (\%) & $3(12.5)$ \\
\hline Length of Stay median (interquartile range) & $7.5(3-12)$ \\
\hline
\end{tabular}

Table 8: Compliance with each element of the sepsis six care bundle

\begin{tabular}{lll}
\hline Therapy & $\begin{array}{l}\text { Achieved within } \\
1 \text { hour } n(\%)\end{array}$ & $\begin{array}{l}\text { Achieved at any } \\
\text { point } n(\%)\end{array}$ \\
\hline IV fluids & $14(58.3)$ & $18(75)$ \\
IV antibiotics & $14(58.3)$ & $19(79.2)$ \\
Oxygen & $5(20.8)$ & $9(37.5)$ \\
Lactate measured & $12(50.0)$ & $17(70.8)$ \\
Blood cultures taken & $5(20.8)$ & $8(33.3)$ \\
Urine output meas- & $4(16.7)$ & $6(25.0)$ \\
ured & & $1(4.17)$ \\
All six & $1(4.17)$ & \\
\hline
\end{tabular}

Sepsis Six Care Bundle. This suggests a certain level of awareness amongst clinicians of the importance of these aspects of the care bundle. However, compliance for these did not meet expected standards in either hospital. Clinicians may have been aware of the lack of evidence of efficacy for certain elements of the Bundle, such as giving oxygen and measuring urine output; both of these interventions had the lowest levels of compliance. ${ }^{13}$

It is not possible to know from this study why compliance with the Sepsis Six Bundle was low. Research in this area suggests lack of compliance with care bundles is multifactorial, and includes 
issues such as quick turnover of medical staff who are not familiar with the care bundle, lack of senior doctor involvement, poor communication and practical barriers such as equipment not being readily available. ${ }^{14-15}$ Improving compliance to care bundles can be difficult because of the multiple factors involved. Several projects have used a combination of education, checklists and stickers, sepsis "champions" and sepsis "packs". 14-17 These combinations of interventions are labour intensive and require sustained implementation to work. A systematic analysis of the effect of performance improvement programmes on compliance with sepsis bundles found that education and process change can successfully improve compliance, and showed a concomitant reduction in mortality. ${ }^{18}$ Quality improvement initiatives in Brazil have reduced hospital mortality from sepsis, however this reduction in mortality resulted from earlier recognition of sepsis, rather than increased compliance to the six-hour sepsis bundle. ${ }^{19}$

Our results demonstrate that sepsis is a common problem, affecting nearly $10 \%$ of acute hospital admissions. There is limited data on the true prevalence of sepsis outside of critical care, and this snapshot of prevalence is one of the few studies to address this. ${ }^{20,21}$ The prevalence of sepsis was higher than the recent studies in Wales that identified that $4.2 \%-5.5 \%$ of in-patients had sepsis depending on the clinical criteria used. ${ }^{7,22-24}$ The most common source of sepsis was pulmonary, which correlates with previous studies that have identified this as the commonest source of infection in patients with sepsis presenting to hospital. ${ }^{22-24}$

In our study, fewer patients had a positive qSOFA score than patients who met the SIRS criteria for sepsis. The intention of qSOFA was to identify patients at risk of the more severe forms of sepsis that have higher mortality rates ${ }^{25}$ and this may explain why qSOFA identified less patients than the SIRS criteria; out of the three patients who died, two met the qSOFA criteria. The qSOFA score was found to be neither sensitive nor specific in a recent UK ward-based study. ${ }^{23,24}$ Similarly, a recent analysis of a large US database failed to confirm the superiority of qSOFA to NEWS in predicting adverse outcomes in patients outside critical care. ${ }^{26} \mathrm{~A}$ comparison of the Sepsis-2 SIRS-related severe sepsis definition to the Sepsis-3 sepsis definition found that they identified a similar cohort of patients with $92 \%$ overlap in the critical care population. ${ }^{27}$ However, this cohort did not include ward patients. More research is needed to establish how best to screen patients for sepsis and identify those at risk of mortality from sepsis.

A particular strength of this study was the use of hand held electronic devices to collect data, which required minimal training, allowed quick data collection and instant upload. This saved time replicating data collected on paper data collection forms, and allowed for standardisation of data collection. The toolkit can be easily adapted for use in other studies. ${ }^{11}$

There are limitations to this study. It was designed to provide a snapshot of the management of patients with suspected sepsis. The study recruited a small number of patients over a short time period in only two centres. Only new admissions with sepsis were identified, missing patients who develop sepsis whilst in hospital. The small number of patients recruited within a short time period makes it difficult for the results to be generalizable. Due to the limited duration of study recruitment, it is likely that only the management skills of a small cohort of clinicians will have been scrutinised. It is possible that performing the study on another day with a different set of clinicians could have yielded different results. However, the repeated nationwide point-prevalence studies in Wales between 2015-2017 have yielded similar results, in incidence of sepsis, bundle compliance and likely cause of death., ${ }^{7,24,27,28}$ Similarly, the international IMPRESS study reported similar rates of sepsis outside of critical care and low bundle compliance. ${ }^{29}$

\section{Conclusions}

The results of this study suggest that sepsis is common, yet management remains sub optimal. Investigation into potential barriers to recognition and management of sepsis can ensure improvements to patient care can be appropriately targeted. It will also be beneficial to establish the true prevalence of sepsis in UK hospitals to help determine the burden of sepsis on the healthcare system and society.

\section{Acknowledgements}

With thanks to the Welsh Intensive Care Society for the provision of the electronic devices and dataset used for data collection.

With thanks to our data collectors: Jo Alderman, Cat Atkin, Maria Benham, Lloyd Cottrell, Amar Jessel, Jamie Mayers, Greg Packer and Vanisha Patel.

\section{References}

1. Singer M, Deutschman CS, Seymour CW, Shankar-Hari M, Annane $D$, Bauer M, et al. The Third International Consensus Definitions for Sepsis and Septic Shock (Sepsis-3). JAMA 2016; 315: 801-810.

2. York Health Economics Consortium. Whitewater charitable trust. The cost of sepsis care in the UK. Final Report 2017. Available from: http:// allcatsrgrey.org.uk/wp/download/health_economics/YHEC-SepsisReport-17.02.17-FINAL.pdf Accessed 25th October 2017.

3. Iwashyna TJ, Cooke CR, Wunsch H, Kahn JM. Population burden of long-term survivorship after severe sepsis in older Americans. J Am Geriatr Soc 2012; 60: 1070-1077.

4. Vincent J-L, Marshall JC, Namendys-Silva SA, Francois B, MartinLoeches I, Lipman J, et al; ICON Investigators. Assessment of the worldwide burden of critical illness: the Intensive Care Over Nations (ICON) audit. Lancet Respir Med 2014;2:380-386.

5. Daniels R, Nutbeam T, McNamara G, Galvin C. The sepsis six and the severe sepsis resuscitation bundle: a prospective observational cohort study. Emerg Med J 2011; 28:507-512.

6. Fletcher SJ, Quinn AC. The Surviving Sepsis Campaign and Sepsis Care Bundles: substance or sophistry? Anaesthesia 2006;61:313-315.

7. Szakmany T, Lundin RM, Sharif B, Ellis G, Morgan P, Kopczynska $M$ et al. Sepsis Prevalence and Outcome on the General Wards and Emergency Departments in Wales: Results of a Multi-Centre, Observational, Point Prevalence Study. PLoS ONE (online) 2016;11: e0167230.

8. Parliamentary and Health Service Ombudsman. Time to Act Severe Sepsis: rapid diagnosis and treatment saves lives. September 2013. Available from: https://www.ombudsman.org.uk/sites/default/ files/2016-08/FINAL_Sepsis_Report_web.pdf Accessed 20th July 2017.

9. National Confidential Enquiry into Patient Outcome and Death. Just Say Sepsis! A review of the process of care received by patient with sepsis. 2015. Available from: http://www.ncepod.org.uk/2015report2/ downloads/JustSaySepsis_FullReport.pdf Accessed 20th July 2017.

10. NHS Health Research Authority. Is my Study Research? Available from: http://www.hra-decisiontools.org.uk/research/ Accessed 20 ${ }^{\text {th }}$ May 2016.

11. Dellinger RP, Levy MM, Rhodes A, Annane D, Gerlach H, Opal SM, et al. Surviving sepsis campaign: international guidelines for management of severe sepsis and septic shock: 2012. Crit Care Med 2013;41:580-637.

12. Sharif B, Lundin RM, Morgan P, Hall JE, Dhadda A, Mann C, et al. Developing a digital data collection platform to measure the prevalence of sepsis in Wales. J Am Med Inform Assoc 2016; 23:11851189. 
13. Vincent J-L, Taccone FS, He X. Harmful Effects of Hyperoxia in Postcardiac Arrest, Sepsis, Traumatic Brain Injury, or Stroke: The Importance of Individualized Oxygen Therapy in Critically III Patients. Can Respir J 2017;2017:2834956.

14. Pinnington S, Atterton B, Ingleby S. Making the journey safe: recognising and responding to severe sepsis in accident and emergency. BMJ Open Qual 2016;5:u210706.w4335.

15. Kumar P, Jordan M, Caesar J, Miller S. Improving the management of sepsis in a district general hospital by implementing the 'Sepsis Six' recommendations. BMJ Qual Improv Rep 2016; 5: u206760.w3983.

16. Bentley J, Henderson S, Thakore S, Donald M, Wang W. Seeking Sepsis in the Emergency Department - Identifying Barriers to Delivery of the Sepsis 6. BMJ Qual Improv Rep 2016;5:u206760.w3983.

17. Burke J, Wood S, Hermon A, Szakmany T. Improving outcome of sepsis on the ward: introducing the "Sepsis Six" bundle. Nurs Crit Care. 2019;24:33-39.

18. Damiani E, Donati A, Serafini G, Rinaldi L, Adrario E, Pelaia P, et al. Effect of performance improvement programs on compliance with sepsis bundles and mortality: a systematic review and meta-analysis of observational studies. PLoS ONE 2015;10:e0125827.

19. Machado FR, Ferreira EM, Schippers P, de Paula IC, Saes LSV, de Oliviera Fl, et al. Implementation of sepsis bundles in public hospitals in Brazil: a prospective study with heterogeneous results. Crit Care 2017;21:2.68.

20. Esteban A, Frutos-Vivar F, Ferguson ND, Peñuelas O, Lorente JA, Gordo $F$, et al. Sepsis incidence and outcome: contrasting the intensive care unit with the hospital ward. Crit Care Med. 2007;35:1284-1289.

21. Rohde JM, Odden AJ, Bonham C, Kuhn L, Malani PN, Chen LM, et al. The epidemiology of acute organ system dysfunction from severe sepsis outside of the intensive care unit. J Hosp Med 2013;8:243-247.

22. Szakmany $T$, Ellis $G$, Lundin RM, Pignatelli I, Sharif B, Joshi $S$, et al. Sepsis in Wales on the general wards: results of a feasibility pilot. $\mathrm{Br}$ J Anaesth 2015;114:1000-1001.
23. Szakmany $T$, Pugh R, Kopczynska M, Lundin RM, Sharif B, Morgan $P$ et al. Defining sepsis on the wards: results of a multi-centre pointprevalence study comparing two sepsis definitions. Anaesthesia 2018;73:195-204.

24. Kopczynska M, Sharif B, Cleaver S, Spencer N, Kurani A, Lee C, et al. Red-flag sepsis and SOFA identifies different patient population at risk of sepsis-related deaths on the general ward. Medicine; 2018;97:e13238.

25. Seymour CW, Liu VX, Iwashyna TJ, Brunkhorst FM, Rea TD, Scherag et al. Assessment of Clinical Criteria for Sepsis: For the Third International Consensus Definitions for Sepsis and Septic Shock (Sepsis-3). JAMA 2016:315:762-774.

26. Churpek MM, Snyder A, Han X Sokol S, Pettit N, Howell MD, et al. Quick Sepsis-related Organ Failure Assessment, Systemic Inflammatory Response Syndrome, and Early Warning Scores for Detecting Clinical Deterioration in Infected Patients outside the Intensive Care Unit. Am J Respir Crit Care Med 2017;195:906-911.

27. Shankar-Hari M, Harrison DA, Rubenfeld GD, Rowan K. Epidemiology of sepsis and septic shock in critical care units: comparison between sepsis-2 and sepsis-3 populations using a national critical care database. Br J Anaesth 2017; 119: 626-636.

28. Kopczynska M, Sharif B, Cleaver S, Spencer N, Kurani A, Lee C, et al. Sepsis-related deaths in the at-risk population on the wards: attributable fraction of mortality in a large point-prevalence study. BMC Res Notes; 2018;11(1):720.

29. Rhodes A, Phillips G, Beale R, Cecconi M, Chiche JD, De Backer D, et al. The Surviving Sepsis Campaign bundles and outcome: results from the International Multicentre Prevalence Study on Sepsis (the IMPreSS study). Intensive Care Med 2015;41:1620-1628. 